PROCEEDINGS OF THE

AMERICAN MATHEMATICAL SOCIETY

Volume 137, Number 7, July 2009, Pages 2225-2232

S 0002-9939(08)09785-2

Article electronically published on December 31, 2008

\title{
GLUING AND HILBERT FUNCTIONS OF MONOMIAL CURVES
}

\author{
FEZA ARSLAN, PINAR METE, AND MESUT ŞAHIN
}

(Communicated by Bernd Ulrich)

\begin{abstract}
In this article, by using the technique of gluing semigroups, we give infinitely many families of 1-dimensional local rings with non-decreasing Hilbert functions. More significantly, these are local rings whose associated graded rings are not necessarily Cohen-Macaulay. In this sense, we give an effective technique for constructing large families of 1-dimensional Gorenstein local rings associated to monomial curves, which support Rossi's conjecture saying that every Gorenstein local ring has a non-decreasing Hilbert function.
\end{abstract}

\section{INTRODUCTION}

In this article, we study the Hilbert functions of local rings associated to affine monomial curves obtained by using the technique of gluing numerical semigroups. The concept of gluing was introduced by J.C. Rosales in 11 and used by several authors to produce new examples of set-theoretic and ideal-theoretic complete intersection affine or projective varieties (for example [10, 12, 13]). We give large families of local rings with non-decreasing Hilbert functions and generalize the results in [1] and 2] given for nice extensions, which are in fact special types of gluings. In doing this, we also give the definition of a nice gluing, which is a generalization of a nice extension. Moreover, by using the technique of nice gluing, we obtain infinitely many families of 1-dimensional local rings with non-CohenMacaulay associated graded rings and still having non-decreasing Hilbert functions. We demonstrate that nice gluing is an effective technique for constructing large families of 1-dimensional Gorenstein local rings associated to monomial curves, which support the conjecture due to Rossi saying that every Gorenstein local ring has non-decreasing Hilbert function [2].

Our main interest in this article is the following question about gluing:

Question. If the Hilbert functions of the local rings associated to two monomial curves are non-decreasing, is the Hilbert function of the local ring associated to the monomial curve obtained by gluing these two monomial curves also non-decreasing?

Every monomial curve in affine 2-space is obtained by gluing, and it is wellknown that every local ring associated to a monomial curve in affine 2-space has a non-decreasing Hilbert function. In affine 3-space, not every monomial curve is

Received by the editors July 17, 2008, and, in revised form, September 19, 2008.

2000 Mathematics Subject Classification. Primary 13H10, 14H20; Secondary $13 \mathrm{P} 10$.

Key words and phrases. Hilbert function of local ring, tangent cone, monomial curve, numerical semigroup, semigroup gluing, nice gluing, Rossi's conjecture.

(C)2008 American Mathematical Society Reverts to public domain 28 years from publication 
obtained by gluing, but every local ring associated to a monomial curve in affine 3 -space also has a non-decreasing Hilbert function. This follows from the important result of Elias saying that every one-dimensional Cohen-Macaulay local ring with embedding dimension three has a non-decreasing Hilbert function [6]. Thus, the above question is trivial for the monomial curves in affine 2 -space and 3-space which are obtained by gluing, while the question is open even for the monomial curves in 4 -space which are obtained by gluing. What makes this question important is that if the answer is affirmative even in the case of gluing complete intersection monomial curves, it will follow that the Hilbert function of every local ring associated to a complete intersection monomial curve is non-decreasing. This would be due to a result of Delorme [4, which is restated by Rosales in terms of gluing and says that every complete intersection numerical semigroup minimally generated by at least two elements is a gluing of two complete intersection numerical semigroups [11, Theorem 2.3]. Considering that it is still not known whether the Hilbert function of local rings with embedding dimension four associated to complete intersection monomial curves in affine 4-space is non-decreasing, this would be an important step in proving Rossi's conjecture.

We recall that an affine monomial curve $C\left(n_{1}, \ldots, n_{k}\right)$ is a curve with generic zero $\left(t^{n_{1}}, \ldots, t^{n_{k}}\right)$ in the affine $n$-space $\mathbb{A}^{n}$ over an algebraically closed field $K$, where $n_{1}<\cdots<n_{k}$ are positive integers with $\operatorname{gcd}\left(n_{1}, n_{2}, \ldots, n_{k}\right)=1$ and $\left\{n_{1}, n_{2}, \ldots, n_{k}\right\}$ is a minimal set of generators for the numerical semigroup $\left\langle n_{1}, n_{2}, \ldots, n_{k}\right\rangle=$ $\left\{n \mid n=\sum_{i=1}^{k} a_{i} n_{i}, a_{i}\right.$ 's are non-negative integers $\}$. The local ring associated to the monomial curve $C=C\left(n_{1}, \ldots, n_{k}\right)$ is $K\left[\left[t^{n_{1}}, \ldots, t^{n_{k}}\right]\right]$, and the Hilbert function of this local ring is the Hilbert function of its associated graded ring $g r_{m}\left(K\left[\left[t^{n_{1}}, \ldots, t^{n_{k}}\right]\right]\right)$, which is isomorphic to the ring $K\left[x_{1}, \ldots, x_{k}\right] / I(C)_{*}$, where $I(C)$ is the defining ideal of $C$ and $I(C)_{*}$ is the ideal generated by the polynomials $f_{*}$, with $f$ in $I(C)$ and $f_{*}$ being the homogeneous summand of $f$ of least degree. In other words, $I(C)_{*}$ is the defining ideal of the tangent cone of $C$ at 0 .

\section{Technique of GLuing Semigroups and monomial CuRves}

In this section, we first give the definition of gluing for numerical semigroups.

Definition 2.1 ([11, Lemma 2.2]). Let $S_{1}$ and $S_{2}$ be two numerical semigroups minimally generated by $m_{1}<\cdots<m_{l}$ and $n_{1}<\cdots<n_{k}$ respectively. Let $p=$ $b_{1} m_{1}+\cdots+b_{l} m_{l} \in S_{1}$ and $q=a_{1} n_{1}+\cdots+a_{k} n_{k} \in S_{2}$ be two positive integers satisfying $\operatorname{gcd}(p, q)=1$ with $p \notin\left\{m_{1}, \ldots, m_{l}\right\}, q \notin\left\{n_{1}, \ldots, n_{k}\right\}$ and $\left\{q m_{1}, \ldots, q m_{l}\right\} \cap$ $\left\{p n_{1}, \ldots, p n_{k}\right\}=\emptyset$. The numerical semigroup $S=\left\langle q m_{1}, \ldots, q m_{l}, p n_{1}, \ldots, p n_{k}\right\rangle$ is called a gluing of the semigroups $S_{1}$ and $S_{2}$.

Thus, the monomial curve $C=C\left(q m_{1}, \ldots, q m_{l}, p n_{1}, \ldots, p n_{k}\right)$ can be interpreted as the gluing of the monomial curves $C_{1}=C\left(m_{1}, \ldots, m_{l}\right)$ and $C_{2}=C\left(n_{1}, \ldots, n_{k}\right)$, if $p$ and $q$ satisfy the conditions in Definition 2.1, Moreover, from [11, Theorem 1.4], if the defining ideals $I\left(C_{1}\right) \subset K\left[x_{1}, \ldots, x_{l}\right]$ of $C_{1}$ and $I\left(C_{2}\right) \subset K\left[y_{1}, \ldots, y_{k}\right]$ of $C_{2}$ are generated by the sets $G_{1}=\left\{f_{1}, \ldots, f_{s}\right\}$ and $G_{2}=\left\{g_{1}, \ldots, g_{t}\right\}$ respectively, then the defining ideal of $I(C) \subset K\left[x_{1}, \ldots, x_{l}, y_{1}, \ldots, y_{k}\right]$ is generated by the set $G=\left\{f_{1}, \ldots, f_{s}, g_{1}, \ldots, g_{t}, x_{1}^{b_{1}} \ldots x_{l}^{b_{l}}-y_{1}^{a_{1}} \ldots y_{k}^{a_{k}}\right\}$.

Now, consider the local rings $R_{1}=K\left[\left[t^{m_{1}}, \ldots, t^{m_{l}}\right]\right], R_{2}=K\left[\left[t^{n_{1}}, \ldots, t^{n_{k}}\right]\right]$ and $R=K\left[\left[t^{q m_{1}}, \ldots, t^{q m_{l}}, t^{p n_{1}}, \ldots, t^{p n_{k}}\right]\right]$ associated respectively to the monomial curves $C_{1}, C_{2}$ and $C$ obtained by gluing $C_{1}$ and $C_{2}$. Our main interest is whether 
the Hilbert function of $R$ is non-decreasing given that the Hilbert functions of the local rings $R_{1}$ and $R_{2}$ are non-decreasing.

We first answer the following question: If $C_{1}$ and $C_{2}$ have Cohen-Macaulay tangent cones, is the tangent cone of the monomial curve $C$ obtained by gluing these two monomial curves necessarily Cohen-Macaulay? The following example shows that the answer is no.

Example 2.2. Let $C_{1}$ and $C_{2}$ be the monomial curves $C_{1}=C(5,12)$ and $C_{2}=$ $C(7,8)$. Obviously, they have Cohen-Macaulay tangent cones. By a gluing of $C_{1}$ and $C_{2}$, we obtain the monomial curve $C=C(21 \times 5,21 \times 12,17 \times 7,17 \times 8)$. The ideal $I(C)$ is generated by the following set $G=\left\{x_{1}^{12}-x_{2}^{5}, y_{1}^{8}-y_{2}^{7}, x_{1} x_{2}-y_{1}^{3}\right\}$. The ideal $I(C)_{*}$ of the tangent cone of $C$ at the origin is generated by the set $G_{*}=$ $\left\{x_{1} x_{2}, x_{2}^{5}, y_{1}^{15}, y_{2}^{7}, x_{2}^{4} y_{1}^{3}, x_{2}^{3} y_{1}^{6}, x_{2}^{2} y_{1}^{9}, x_{2} y_{1}^{12}\right\}$ which is a Gröbner basis with respect to the negative degree reverse lexicographical ordering with $x_{2}>y_{2}>y_{1}>x_{1}$. From [1, Theorem 2.1], since $x_{1}$ divides $x_{1} x_{2} \in G_{*}$, the monomial curve $C$ obtained by a gluing of $C_{1}$ and $C_{2}$ does not have a Cohen-Macaulay tangent cone. It should also be noted that the Hilbert function of the local ring corresponding to $C$ is non-decreasing, although the tangent cone of $C$ is not Cohen-Macaulay.

This example leads us to ask the following question:

Question. If two monomial curves have Cohen-Macaulay tangent cones, under what conditions does the monomial curve obtained by gluing these two monomial curves also have a Cohen-Macaulay tangent cone?

To answer this question partially, we first give the definition of a nice gluing, which generalizes the definition of a nice extension given in [2].

Definition 2.3. Let $S_{1}=\left\langle m_{1}, \ldots, m_{l}\right\rangle$ and $S_{2}=\left\langle n_{1}, \ldots, n_{k}\right\rangle$ be two numerical semigroups minimally generated by $m_{1}<\cdots<m_{l}$ and $n_{1}<\cdots<n_{k}$ respectively. The numerical semigroup $S=\left\langle q m_{1}, \ldots, q m_{l}, p n_{1}, \ldots, p n_{k}\right\rangle$ obtained by gluing $S_{1}$ and $S_{2}$ is called a nice gluing if $p=b_{1} m_{1}+\cdots+b_{l} m_{l} \in S_{1}$ and $q=a_{1} n_{1} \in S_{2}$ with $a_{1} \leq b_{1}+\cdots+b_{l}$.

Remark 2.4. Notice that a nice extension as defined in [2] is exactly a nice gluing with $S_{2}=\langle 1\rangle$.

Remark 2.5. It is important to determine the smallest integer among the generators of the numerical semigroup $S=\left\langle q m_{1}, \ldots, q m_{l}, p n_{1}, \ldots, p n_{k}\right\rangle$ obtained by gluing, since this is essential in checking the Cohen-Macaulayness of the tangent cone of the associated monomial curve. The condition $a_{1} \leq b_{1}+\cdots+b_{l}$ with $m_{1}<\cdots<m_{l}$, $n_{1}<\cdots<n_{k}, \operatorname{gcd}(p, q)=1$ and $\left\{q m_{1}, \ldots, q m_{l}\right\} \cap\left\{p n_{1}, \ldots, p n_{k}\right\}=\emptyset$ implies that

$$
q m_{1}=a_{1} n_{1} m_{1} \leq\left(b_{1}+\cdots+b_{l}\right) n_{1} m_{1}<p n_{1}=\left(b_{1} m_{1}+\cdots+b_{l} m_{l}\right) n_{1}
$$

and that $q m_{1}$ is the smallest integer among the generators of $S$.

We can now state the following theorem:

Theorem 2.6. Let $S_{1}=\left\langle m_{1}, \ldots, m_{l}\right\rangle$ and $S_{2}=\left\langle n_{1}, \ldots, n_{k}\right\rangle$ be two numerical semigroups minimally generated by $m_{1}<\cdots<m_{l}$ and $n_{1}<\cdots<n_{k}$, and let $S=\left\langle q m_{1}, \ldots, q m_{l}, p n_{1}, \ldots, p n_{k}\right\rangle$ be a nice gluing of $S_{1}$ and $S_{2}$. If the associated monomial curves $C_{1}=C\left(m_{1}, \ldots, m_{l}\right)$ and $C_{2}=C\left(n_{1}, \ldots, n_{k}\right)$ have CohenMacaulay tangent cones at the origin, then $C=C\left(q m_{1}, \ldots, q m_{l}, p n_{1}, \ldots, p n_{k}\right)$ also 
has a Cohen-Macaulay tangent cone at the origin, and thus the Hilbert function of the local ring $K\left[\left[t^{q m_{1}}, \ldots, t^{q m_{l}}, t^{p n_{1}}, \ldots, t^{p n_{k}}\right]\right]$ is non-decreasing.

To prove this theorem, we first give a refinement of the criterion for checking the Cohen-Macaulayness of the tangent cone of a monomial curve given in 1, Theorem 2.1], which was used in Example 2.2. The advantage of this modification in the criterion is that instead of first finding the generators of the tangent cone and then computing another Gröbner basis, it only needs the computation of the standard basis of the generators of the defining ideal of the monomial curve with respect to a special local order. Recall that a local order is a monomial ordering with 1 greater than any other monomial. For examples and properties of local orderings, see $[8$. We denote the leading monomial of a polynomial $f$ by $\operatorname{LM}(f)$.

Lemma 2.7. Let $\left\langle n_{1}, \ldots, n_{k}\right\rangle$ be a numerical semigroup minimally generated by $n_{1}<\cdots<n_{k}$, let $C=C\left(n_{1}, \ldots, n_{k}\right)$ be the associated monomial curve and let $G=\left\{f_{1}, \ldots, f_{s}\right\}$ be a minimal standard basis of the ideal $I(C) \subset K\left[x_{1}, \ldots, x_{k}\right]$ with respect to the negative degree reverse lexicographical ordering that makes $x_{1}$ the lowest variable. $C$ has Cohen-Macaulay tangent cone at the origin if and only if $x_{1}$ does not divide $\operatorname{LM}\left(f_{i}\right)$ for $1 \leq i \leq s$.

By using a local ordering, this lemma combines a result of Bayer-Stillman [5, Theorem 15.13] with the well-known fact that a monomial curve $C=C\left(n_{1}, \ldots, n_{k}\right)$, where $n_{1}$ is smallest among the integers $n_{1}, \ldots, n_{k}$, has Cohen-Macaulay tangent cone if and only if $x_{1}$ is not a zero-divisor in the ring $K\left[x_{1}, \ldots, x_{k}\right] / I(C)_{*}$, 7 , Theorem 7].

Proof. Recalling that $f_{*}$ is the homogeneous summand of the polynomial $f$ of least degree, if $x_{1}$ divides $\operatorname{LM}\left(f_{i}\right)$ for some $i$, then either $f_{i_{*}}=x_{1} m$ or $f_{i_{*}}=x_{1} m+$ $\sum c_{i} m_{i}$, where the $m_{i}$ 's are monomials having the same degree as $x_{1} m$ and the $c_{i}$ 's are in $K$. In the latter case, $x_{1}$ must divide each $m_{i}$, because we work with the negative degree reverse lexicographical ordering that makes $x_{1}$ the lowest variable. This implies that in both cases $f_{i_{*}}=x_{1} g$ where $g$ is a homogeneous polynomial. Moreover, $g \notin I(C)_{*}$. If $g \in I(C)_{*}$, then there exists $f \in I(C)$ such that $f_{*}=$ $g$, so $\operatorname{LM}(f)=\operatorname{LM}(g)$. Since $\left\langle\operatorname{LM}\left(f_{1}\right), \ldots, \operatorname{LM}\left(f_{s}\right)\right\rangle=\langle\operatorname{LM}(I(C))\rangle$, there exists an $f_{j} \in G$ such that $\operatorname{LM}\left(f_{j}\right)$ divides $\operatorname{LM}(f)=\operatorname{LM}(g)$, and this contradicts the minimality of $G$. Thus, $x_{1} g \in I(C)_{*}$, while $g \notin I(C)_{*}$, which makes $x_{1}$ a zerodivisor in $K\left[x_{1}, \ldots, x_{k}\right] / I(C)_{*}$. Hence, the tangent cone of the monomial curve $C$ is not Cohen-Macaulay. Conversely, if $K\left[x_{1}, \ldots, x_{k}\right] / I(C)_{*}$ is not Cohen-Macaulay, then $x_{1}$ is a zero-divisor in $K\left[x_{1}, \ldots, x_{k}\right] / I(C)_{*}$. Thus, $x_{1} g \in I(C)_{*}$, where $g$ is a monomial or a homogeneous polynomial with $g \notin I(C)_{*}$, so that $\operatorname{LM}\left(f_{i}\right) \nmid \operatorname{LM}(g)$ for $1 \leq i \leq s$. Since the ideal generated by the leading monomials of the elements in $I(C)$ obviously contains $x_{1} \operatorname{LM}(g)$, there exists $f_{i} \in G$ such that $\operatorname{LM}\left(f_{i}\right)=x_{1} m$, where $m$ is a monomial that divides $\operatorname{LM}(g)$. This completes the proof.

We can now prove Theorem 2.6.

Proof of Theorem 2.6. By using the notation in [8], we denote the s-polynomial of the polynomials $f$ and $g$ by $\operatorname{spoly}(f, g)$ and Mora's polynomial weak normal form of $f$ with respect to $G$ by $N F(f \mid G)$. Let $G_{1}=\left\{f_{1}, \ldots, f_{s}\right\}$ be a minimal standard basis of the ideal $I\left(C_{1}\right) \subset K\left[x_{1}, \ldots, x_{l}\right]$ with respect to the negative degree reverse lexicographical ordering with $x_{2}>\cdots>x_{l}>x_{1}$, and let $G_{2}=\left\{g_{1}, \ldots, g_{t}\right\}$ be a minimal standard basis of the ideal $I\left(C_{2}\right) \subset K\left[y_{1}, \ldots, y_{k}\right]$ with respect to the 
negative degree reverse lexicographical ordering with $y_{2}>\cdots>y_{k}>y_{1}$. Since $C_{1}$ and $C_{2}$ have Cohen-Macaulay tangent cones at the origin, we conclude from Lemma 2.7 that $x_{1}$ does not divide the leading monomial of any element in $G_{1}$ and $y_{1}$ does not divide the leading monomial of any element in $G_{2}$ for the given orderings. The defining ideal of the monomial curve $C$ obtained by gluing is generated by the set $G=\left\{f_{1}, \ldots, f_{s}, g_{1}, \ldots, g_{t}, x_{1}^{b_{1}} \ldots x_{l}^{b_{l}}-y_{1}^{a_{1}}\right\}$. Moreover, this set is a minimal standard basis with respect to the negative degree reverse lexicographical ordering with $y_{2}>\cdots>y_{k}>y_{1}>x_{2}>\cdots>x_{l}>x_{1}$, because $N F\left(\operatorname{spoly}\left(f_{i}, g_{j}\right) \mid G\right)=0$, $N F\left(\operatorname{spoly}\left(f_{i}, x_{1}^{b_{1}} \ldots x_{l}^{b_{l}}-y_{1}^{a_{1}}\right) \mid G\right)=0$ and $N F\left(\operatorname{spoly}\left(g_{j}, x_{1}^{b_{1}} \ldots x_{l}^{b_{l}}-y_{1}^{a_{1}}\right) \mid G\right)=0$ for $1 \leq i \leq s$ and $1 \leq j \leq t$. This is due to the fact that $N F(\operatorname{spoly}(f, g) \mid G)=0$ if $\operatorname{lcm}(\operatorname{LM}(f), \operatorname{LM}(g))=\operatorname{LM}(f) \cdot \operatorname{LM}(g)$. From Remark 2.5. $q m_{1}$ is the smallest integer among the generators of $G$. Thus, $C$ has a Cohen-Macaulay tangent cone at the origin if and only if $x_{1}$, which corresponds to $q m_{1}$, is not a zero-divisor in $K\left[x_{1}, \ldots, x_{l}, y_{1}, \ldots, y_{k}\right] / I(C)_{*}$. Since $x_{1}$ does not divide the leading monomial of any element in $G_{1}$ and $G_{2}$, and $\operatorname{LM}\left(x_{1}^{b_{1}} \ldots x_{l}^{b_{l}}-y_{1}^{a_{1}}\right)=y_{1}^{a_{1}}, x_{1}$ does not divide the leading monomial of any element in $G$, which is a minimal standard basis with respect to the negative degree reverse lexicographical ordering with $y_{2}>\cdots>y_{k}>$ $y_{1}>x_{2}>\cdots>x_{l}>x_{1}$. Thus, from Lemma 2.7 $C$ has a Cohen-Macaulay tangent cone at the origin.

Remark 2.8. From Remark 2.4, every nice extension is a nice gluing. Thus, if the monomial curve $C=C\left(m_{1}, \ldots, m_{l}\right)$ has a Cohen-Macaulay tangent cone at the origin, then every monomial curve $C^{\prime}=C\left(q m_{1}, \ldots, q m_{l}, b_{1} m_{1}+\cdots+b_{l} m_{l}\right)$ obtained by a nice gluing also has a Cohen-Macaulay tangent cone at the origin. Thus, Theorem 2.6 generalizes the results in [1, Proposition 4.1] and [2, Theorem 3.6].

Example 2.9. Let $C_{1}$ and $C_{2}$ be the monomial curves $C_{1}=C\left(m_{1}, m_{2}\right)$ with $m_{1}<$ $m_{2}$ and $C_{2}=C\left(n_{1}, n_{2}\right)$ with $n_{1}<n_{2}$. Obviously, they have Cohen-Macaulay tangent cones. From Theorem 2.6, every monomial curve $C=C\left(q m_{1}, q m_{2}, p n_{1}, p n_{2}\right)$ obtained by a nice gluing with $q=a_{1} n_{1}, p=b_{1} m_{1}+b_{2} m_{2}, \operatorname{gcd}(p, q)=1$ and $a_{1} \leq b_{1}+b_{2}$ has a Cohen-Macaulay tangent cone at the origin, so the local ring $R=K\left[\left[t^{q m_{1}}, t^{q m_{2}}, t^{p n_{1}}, t^{p n_{2}}\right]\right]$ associated to the monomial curve $C$ has a nondecreasing Hilbert function. Thus, by starting with fixed $m_{1}, m_{2}, n_{1}$ and $n_{2}$, we can construct infinitely many families of 1-dimensional local rings with non-decreasing Hilbert functions. For example, consider the monomial curves $C_{1}=C(2,3)$ and $C_{2}=C(4,5)$. By choosing $q=2 n_{1}=8$ and $p=(2 r) m_{1}+m_{2}=4 r+3$, for any $r \geq 1$, we obtain the monomial curve $C(16,24,16 r+12,20 r+15)$, which is a nice gluing of $C_{1}$ and $C_{2}$. Since $C$ is also a complete intersection monomial curve having a Cohen-Macaulay tangent cone, the associated local rings are Gorenstein with non-decreasing Hilbert functions. Obviously, they support Rossi's conjecture.

This example shows that gluing is an effective method for obtaining new families of monomial curves with Cohen-Macaulay tangent cones. Especially in affine 4space, nice gluing is a very efficient method by which to obtain large families of complete intersection monomial curves with Cohen-Macaulay tangent cones, since every monomial curve in affine 2 -space has a Cohen-Macaulay tangent cone.

\section{Monomial curves with non-Cohen-Macaulay tangent cones}

In this section we show that nice gluing is not only an efficient tool by which to obtain new families of monomial curves with Cohen-Macaulay tangent cones 
but, more significantly, it is also very useful for obtaining families of monomial curves with non-Cohen-Macaulay tangent cones that have non-decreasing Hilbert functions. In other words, it is an effective method for obtaining families of local rings with non-decreasing Hilbert functions. In this sense, it can be used to obtain families of local rings in proving the conjecture due to Rossi which says that a one-dimensional Gorenstein local ring has a non-decreasing Hilbert function.

Theorem 3.1. Let $S_{1}=\left\langle m_{1}, \ldots, m_{l}\right\rangle$ and $S_{2}=\left\langle n_{1}, \ldots, n_{k}\right\rangle$ be two numerical semigroups minimally generated by $m_{1}<\cdots<m_{l}$ and $n_{1}<\cdots<n_{k}$, and let $S=\left\langle q m_{1}, \ldots, q m_{l}, p n_{1}, \ldots, p n_{k}\right\rangle$ be a nice gluing of $S_{1}$ and $S_{2}$. (Recall that $p=b_{1} m_{1}+\cdots+b_{l} m_{l} \in S_{1}$ and $q=a_{1} n_{1} \in S_{2}$ with $a_{1} \leq b_{1}+\cdots+$ $b_{l}$.) Let the local ring $K\left[\left[t^{m_{1}}, \ldots, t^{m_{l}}\right]\right]$ associated to the monomial curve $C_{1}=$ $C\left(m_{1}, \ldots, m_{l}\right)$ have a non-decreasing Hilbert function and let $C_{2}=C\left(n_{1}, \ldots, n_{k}\right)$ have a Cohen-Macaulay tangent cone at the origin; then the Hilbert function of the local ring $K\left[\left[t^{q m_{1}}, \ldots, t^{q m_{l}}, t^{p n_{1}}, \ldots, t^{p n_{k}}\right]\right]$ associated to the monomial curve $C=C\left(q m_{1}, \ldots, q m_{l}, p n_{1}, \ldots, p n_{k}\right)$ obtained by gluing is also non-decreasing.

Proof. Let $G_{1}=\left\{f_{1}, \ldots, f_{s}\right\}$ be a minimal standard basis of the ideal $I\left(C_{1}\right) \subset$ $K\left[x_{1}, \ldots, x_{l}\right]$ with respect to the negative degree reverse lexicographical ordering with $x_{2}>\cdots>x_{l}>x_{1}$, and let $G_{2}=\left\{g_{1}, \ldots, g_{t}\right\}$ be a minimal standard basis of the ideal $I\left(C_{2}\right) \subset K\left[y_{1}, \ldots, y_{k}\right]$ with respect to the negative degree reverse lexicographical ordering with $y_{2}>\cdots>y_{k}>y_{1}$. Since $C_{2}$ has Cohen-Macaulay tangent cone, $y_{1}$ does not divide $\operatorname{LM}\left(g_{i}\right)$ for $1 \leq i \leq t$ by Lemma 2.7. From the proof of Theorem 2.6. $G=\left\{f_{1}, \ldots, f_{s}, g_{1}, \ldots, g_{t}, x_{1}^{b_{1}} \ldots x_{l}^{b_{l}}-y_{1}^{a_{1}}\right\}$ is a minimal standard basis with respect to the negative degree reverse lexicographical ordering with $y_{2}>\cdots>y_{k}>y_{1}>x_{2}>\cdots>x_{l}>x_{1}$, and again from Lemma 2.7 we have $\left\langle\operatorname{LM}\left(I(C)_{*}\right)\right\rangle=\left\langle\operatorname{LM}\left(f_{1}\right), \ldots, \operatorname{LM}\left(f_{s}\right), \operatorname{LM}\left(g_{1}\right), \ldots, \operatorname{LM}\left(g_{t}\right), y_{1}^{a_{1}}\right\rangle$. Hence, recalling the well-known result going back to Macaulay [9], the Hilbert function of the local ring $K\left[\left[t^{q m_{1}}, \ldots, t^{q m_{l}}, t^{p n_{1}}, \ldots, t^{p n_{k}}\right]\right]$ is equal to the Hilbert function of the graded ring

$$
R=K\left[x_{1}, \ldots, x_{l}, y_{1}, \ldots, y_{k}\right] /\left\langle\operatorname{LM}\left(f_{1}\right), \ldots, \operatorname{LM}\left(f_{s}\right), \operatorname{LM}\left(g_{1}\right), \ldots, \operatorname{LM}\left(g_{t}\right), y_{1}^{a_{1}}\right\rangle .
$$

By using [3, Proposition 2.4] and recalling that $y_{1} \nmid \operatorname{LM}\left(g_{i}\right)$ for $1 \leq i \leq t$, one finds that $R$ is isomorphic to $R_{1} \otimes_{K} R_{2} \otimes_{K} R_{3}$, where $R_{1}=K\left[x_{1}, \ldots, x_{l}\right] /\left\langle\operatorname{LM}\left(f_{1}\right), \ldots\right.$, $\left.\operatorname{LM}\left(f_{s}\right)\right\rangle, R_{2}=K\left[y_{2}, \ldots, y_{l}\right] /\left\langle\operatorname{LM}\left(g_{1}\right), \ldots, \operatorname{LM}\left(g_{t}\right)\right\rangle$ and $R_{3}=K\left[y_{1}\right] /\left\langle y_{1}^{a_{1}}\right\rangle$. Moreover, the Hilbert series of $R$ is the product of the Hilbert series of $R_{1}, R_{2}$ and $R_{3}$. The Hilbert series of $R_{1}$ can be given as $h_{1}(t) /(1-t)$, where the polynomial $h_{1}(t)$ has non-negative coefficients, since from the assumption the local ring associated to the monomial curve $C_{1}$ has non-decreasing Hilbert function. $R_{2}$ is Artinian, hence its Hilbert series $h_{2}(t)$ has non-negative coefficients. Observing that the Hilbert series of $R_{3}$ is $h_{3}(t)=1+t+\cdots+t^{a_{1}-1}$, we obtain that the Hilbert series of $R$ is $h_{1}(t) h_{2}(t) h_{3}(t) /(1-t)$, where the polynomial $h_{1}(t) h_{2}(t) h_{3}(t)$ has non-negative coefficients. This proves that the Hilbert function of $R$ is non-decreasing.

We can now use this theorem to obtain large families of Gorenstein monomial curves with non-Cohen-Macaulay tangent cones having non-decreasing Hilbert functions to support Rossi's conjecture.

Example 3.2. Let $C_{1}$ and $C_{2}$ be the monomial curves $C_{1}=C(6,7,15)$ and $C_{2}=$ $C(1) . C_{1}$ has a non-Cohen-Macaulay tangent cone and a non-decreasing Hilbert function. Obviously, $C_{1}$ and $C_{2}$ satisfy the conditions of Theorem 3.1, which implies 
that every local ring associated to the monomial curve $C=C(6 q, 7 q, 15 q, 6 q+7)$ obtained by a nice gluing (which is also a nice extension) with $q \not \equiv 0(\bmod 7)$ has a non-decreasing Hilbert function. $C_{1}$ is a complete intersection monomial curve, with $I\left(C_{1}\right)=\left\langle x_{1}^{5}-x_{3}^{2}, x_{1} x_{3}-x_{2}^{3}\right\rangle$ having a minimal standard basis with respect to the negative degree reverse lexicographical ordering with $x_{2}>x_{3}>x_{1}$ given by $\left\{x_{1}^{5}-x_{3}^{2}, x_{1} x_{3}-x_{2}^{3}, x_{2}^{3} x_{3}-x_{1}^{6}, x_{2}^{6}-x_{1}^{7}\right\}$. Hence, $C$ is a complete intersection monomial curve, with $I(C)=\left\langle x_{1}^{5}-x_{3}^{2}, x_{1} x_{3}-x_{2}^{3}, y_{1}^{q}-x_{1}^{q} x_{2}\right\rangle$ having a minimal standard basis with respect to the negative degree reverse lexicographical ordering with $y_{1}>x_{2}>x_{3}>x_{1}$ given by $\left\{x_{1}^{5}-x_{3}^{2}, x_{1} x_{3}-x_{2}^{3}, x_{2}^{3} x_{3}-x_{1}^{6}, x_{2}^{6}-x_{1}^{7}, y_{1}^{q}-\right.$ $\left.x_{1}^{q} x_{2}\right\}$, which shows that $C$ has a non-Cohen-Macaulay tangent cone. Thus, we have obtained Gorenstein local rings $K\left[\left[t^{6 q}, t^{7 q}, t^{15 q}, t^{6 q+7}\right]\right]$ with $q \not \equiv 0(\bmod 7)$ having a non-Cohen-Macaulay associated graded rings and non-decreasing Hilbert functions. In this way, starting with a complete intersection monomial curve $C_{1}$ in affine 3-space having a non-Cohen-Macaulay tangent cone, we can construct infinitely many families of 1-dimensional Gorenstein local rings with non-CohenMacaulay associated graded rings and non-decreasing Hilbert functions. In this way, we can construct infinitely many families of Gorenstein local rings supporting Rossi's conjecture.

Corollary 3.3. Every local ring with embedding dimension 4 associated to a monomial curve obtained by a nice gluing of

a) $C_{1}=C\left(m_{1}, m_{2}\right)$ with $m_{1}<m_{2}$ and $C_{2}=C\left(n_{1}, n_{2}\right)$ with $n_{1}<n_{2}$,

b) $C_{1}=C\left(m_{1}, m_{2}, m_{3}\right)$ with $m_{1}<m_{2}<m_{3}$ and $C_{2}=C(1)$, or

c) $C_{1}=C(1)$ and $C_{2}=C\left(n_{1}, n_{2}, n_{3}\right)$ with $n_{1}<n_{2}<n_{3}$, whose tangent cone is Cohen-Macaulay

has a non-decreasing Hilbert function.

Proof. In part a), the result follows from both Theorem 2.6 and Theorem 3.1. In part b), the result follows from Theorem 3.1, since every local ring associated to the monomial curve $C_{1}=C\left(m_{1}, m_{2}, m_{3}\right)$ has non-decreasing Hilbert function due to a result of Elias [6]. In the same way, in part c), the result is a direct consequence of Theorem 3.1 .

\section{ACKNowledgements}

We would like to thank Apostolos Thoma and Marcel Morales for mentioning the connection between extension and gluing. We also would like to thank the referee for very helpful suggestions.

\section{REFERENCES}

[1] F. Arslan, Cohen-Macaulayness of tangent cones, Proc. Amer. Math. Soc. 128 (2000) 22432251. MR1653409 (2000k:13021)

[2] F. Arslan, P. Mete, Hilbert functions of Gorenstein monomial curves, Proc. Amer. Math. Soc. 135 (2007) 1993-2002. MR2299471 (2007m:13023)

[3] D. Bayer, M. Stillman, Computation of Hilbert functions, Journal of Symbolic Computation 14 (1992) 31-50. MR1177988 (94f:13018)

[4] C. Delorme, Sous-monoïdes d'intersection complète de $N$, Ann. Sci. École Norm. (4) 9, no. 1 (1976) 145-154. MR0407038 (53:10821)

[5] D. Eisenbud, Commutative Algebra. With a View toward Algebraic Geometry, SpringerVerlag, New York, 1995. MR1322960(97a:13001)

[6] J. Elias, The conjecture of Sally on the Hilbert function for curve singularities, Journal of Algebra 160, no. 1 (1993) 42-49. MR1237076 (94j:13018) 
[7] A. Garcia, Cohen-Macaulayness of the associated graded of a semigroup ring, Comm. Algebra 10, no. 4 (1982) 393-415. MR649344 (83k:13013)

[8] G.-M. Greuel, G. Pfister, A Singular Introduction to Commutative Algebra, Springer-Verlag, Berlin, 2002. MR:1930604 (2003k:13001)

[9] F. S. Macaulay, The Algebraic Theory of Modular Systems, Cambridge University Press, New York, 1916. MR1281612 (95i:13001)

[10] M. Morales, A. Thoma, Complete intersection lattice ideals, Journal of Algebra 284 (2005) 755-770. MR2114578 (2005j:20076)

[11] J.C. Rosales, On presentations of subsemigroups of $\mathbb{N}^{n}$, Semigroup Forum 55 (1997) 152-159. MR:1457760 (98h:20104)

[12] M. Şahin, Producing set-theoretic complete intersection monomial curves in $\mathbb{P}^{n}$, Proc. Amer. Math. Soc. 137 (2009) 1223-1233.

[13] A. Thoma, Construction of set-theoretic complete intersections via semigroup gluing, Contributions to Algebra and Geometry 41(1) (2000) 195-198. MR.1745589 (2001h:14059)

Department of Mathematics, Middle East Technical University, Ankara, 06531, TURKEY

E-mail address: sarslan@metu.edu.tr

Department of Mathematics, Balikesir University, Balikesir, 10145, Turkey

E-mail address: pinarm@balikesir.edu.tr

Department of Mathematics, Atilim University, Ankara, 06836, Turkey

E-mail address: mesutsahin@gmail.com 